\title{
THE LOGARITHMIC GROWTH OF AN ELEMENT OF THE ROBBA RING WHICH SATISFIES A FROBENIUS EQUATION
}

\author{
TAKAHIRO NAKAGAWA
}

(Received October 29, 2010, revised August 8, 2012)

\begin{abstract}
We study the logarithmic growth of an element of the Robba ring which satisfies a Frobenius equation over the bounded Robba ring. Chiarellotto and Tsuzuki computed the logarithmic growth of analytic functions on the open unit disc with coefficients in a $p$-adic local field which satisfy Frobenius equations over bounded functions of rank 2 . We extend their result by replacing those functions by elements of the Robba ring which satisfy Frobenius equations over the bounded Robba ring. Moreover, we will see, in special cases, the zeros of these functions have some cyclicity and the logarithmic growth can be computed by the zeros of these function.
\end{abstract}

1. Introduction. Let $p$ be a prime number and $K$ a complete discrete valued field of mixed characteristic $(0, p)$. We denote by || the norm of the algebraic closure $\bar{K}$ of $K$ which is normalized by $|p|=p^{-1}$. Let $\mathcal{A}[0,1)$ be the ring of power series with coefficients in $K$ which are convergent in the open unit disc. Let $K[[x]]_{0}$ be the ring of power series which are bounded in the open unit disc. For $f \in \mathcal{A}[0,1)$, the log-growth of $f$ is the growth of coefficients of $f$ (cf. Definition 2.8).

The log-growth of the solutions of differential equations over $K[[x]]_{0}$ was studied by Dwork [8], [9], Robba, [14], and Christol [6]. Especially, Dwork computed the log-growth of the solutions of hypergeometric differential equations, using the fact that they have Frobenius structures [9].

Generally, the log-growth of horizontal sections of a differential module over $K[[x]]_{0}$ with Frobenius structure was not known very well. But recently, Chiarellotto and Tsuzuki showed that the log-growth polygon coincides with the Frobenius polygon in their cases by direct computations [4], [5]. In their proof [4], Chiarellotto and Tsuzuki computed the loggrowth of $f \in \mathcal{A}[0,1)$ when $f$ satisfies a Frobenius equation

$$
a_{0} f+a_{1} f^{\phi}+a_{2} f^{\phi^{2}}=0\left(a_{i} \in K[[x]]_{0}, a_{0} \neq 0\right),
$$

where $\phi\left(\sum_{n \geq 0} a_{n} x^{n}\right)=\sum_{n \geq 0} a_{n} x^{p n}$.

In this paper, we consider a Frobenius equation

$$
a_{0} f+a_{1} f^{\phi}+\cdots+a_{n} f^{\phi^{n}}=0\left(a_{i} \in \mathcal{R}_{0}, a_{0} \neq 0\right),
$$

2000 Mathematics Subject Classification. Primary 12H25.

Key words and phrases. Logarithmic growth, $p$-adic differential equation.

Partly supported by the Grant-in-Aid for Scientific Research (B), Japan Society for the Promotion of Science. 
where $\mathcal{R}_{0}$ is the bounded Robba ring furnished with the Gauss norm ||$_{1}$ which will be defined in Subsection 2.4 (see [4, Propositions 7.6, 7.7]). As a result, we will have the following theorem.

THEOREM 1.1. Let $f$ be a nonzero element of the Robba ring $\mathcal{R}$ which satisfies a Frobenius equation

$$
a_{0} f+a_{1} f^{\phi}+a_{2} f^{\phi^{2}}+\cdots+a_{n} f^{\phi^{n}}=0 \quad\left(a_{i} \in \mathcal{R}_{0}, a_{0} \neq 0\right) .
$$

Suppose that $a_{0}, \ldots, a_{n}$ satisfy the condition

(a) $\left|a_{1} / a_{0}\right|_{1}>\left|a_{2} / a_{1}\right|_{1}>\cdots>\left|a_{n} / a_{n-1}\right|_{1}$.

Then the limit $\alpha=\lim _{r \rightarrow 1^{-}}\left(\log _{p}|f|_{r}-\log _{p}\left|f^{\phi}\right|_{r}\right)$ exists and the exact order of log-growth of $f$ is $\alpha$. Moreover, $\alpha=\log _{p}\left|a_{i+1} / a_{i}\right|_{1}$ for some $i$.

This value $\alpha$ in the theorem is also equal to one of the slope of the Newton polygon of the polynomial

$$
a_{0}+a_{1} X+\cdots+a_{n} X^{n}
$$

with respect to the Gauss norm. In particular, the order of log-growth of $f$ is rational (see [5]). We also consider the appearance of zeros of $f$ when $f$ satisfies a Frobenius equation (1). If $f \in \mathcal{A}[0,1)$ satisfies the condition (a), then we see, for any $r \in[0,1)$ close enough to 1 , that the difference between the number of zeros of $f$ over the closed disc of radius $r$ and that of $f^{\phi}$ is independent of $r$ (see Example 3.11).

Acknowledgment. The author is grateful to Professor Shigeki Matsuda for his helpful advice.

2. $p$-adic analytic function. In this section, we introduce the notation and review the theory of $p$-adic analytic functions.

2.1. Critical radius and Weierstrass Preparation theorem. Let $p$ be a prime number. Let $K$ be a complete discrete valued field with mixed characteristic $(0, p)$. Let | | be the norm of $K$ normalized by $|p|=p^{-1}$, and extended to an algebraic closure $\bar{K}$ of $K$. We denote the open $\operatorname{disc}\{a \in \bar{K} ;|a|<r\}$ by $D\left(0, r^{-}\right)$. For $0 \leq r_{0} \leq r_{1}$ (resp. $\left.0 \leq r_{0}<r_{1}\right)$, we denote

$$
\begin{aligned}
& C\left[r_{0}, r_{1}\right]=\left\{a \in \bar{K} ; r_{0} \leq|a| \leq r_{1}\right\}, \\
& C\left[r_{0}, r_{1}\right)=\left\{a \in \bar{K} ; r_{0} \leq|a|<r_{1}\right\},
\end{aligned}
$$

and call them the annuli with radii $\left[r_{0}, r_{1}\right]$ (resp. $\left[r_{0}, r_{1}\right)$ ). We denote the ring of analytic functions on $C\left[r_{0}, r_{1}\right]$ (resp. $C\left[r_{0}, r_{1}\right)$ ) with coefficients in $K$ by $\mathcal{A}\left[r_{0}, r_{1}\right]$ (resp. $A\left[r_{0}, r_{1}\right)$ ), i.e.,

$$
\begin{aligned}
& \mathcal{A}\left[r_{0}, r_{1}\right]=\left\{\sum_{n=-\infty}^{\infty} a_{n} x^{n} ; a_{n} \in K, \lim _{n \rightarrow \pm \infty}\left|a_{n}\right| r^{n}=0 \text { for all } r \in\left[r_{0}, r_{1}\right]\right\}, \\
& \mathcal{A}\left[r_{0}, r_{1}\right)=\left\{\sum_{n=-\infty}^{\infty} a_{n} x^{n} ; a_{n} \in K, \lim _{n \rightarrow \pm \infty}\left|a_{n}\right| r^{n}=0 \text { for all } r \in\left[r_{0}, r_{1}\right)\right\} .
\end{aligned}
$$


For each $r \in\left[r_{0}, r_{1}\right], \mathcal{A}\left[r_{0}, r_{1}\right]$ have the norm $\mid{ }_{r}$ defined by

$$
\left|\sum_{n=-\infty}^{\infty} a_{n} x^{n}\right|_{r}:=\sup \left|a_{n}\right| r^{n} .
$$

We denote the ring of bounded power series with coefficients in $K$ by

$$
K[[x]]_{0}=\left\{\sum_{n=0}^{\infty} a_{n} x^{n} \in K[[x]] ; \sup _{0 \leq n<\infty}\left|a_{n}\right|<\infty\right\} .
$$

$K[[x]]_{0}$ has the Gauss norm defined by

$$
\left|\sum_{n=0}^{\infty} a_{n} x^{n}\right|_{1}:=\sup \left|a_{n}\right| .
$$

Let $y$ be an element of $C\left[r_{0}, r_{1}\right]$ and $f=\sum_{-\infty<n<\infty} a_{n} x^{n}$ an element of $\mathcal{A}\left[r_{0}, r_{1}\right]$. When $|y|=r \in\left[r_{0}, r_{1}\right]$, we can easily see $|f(y)| \leq|f|_{r}$. Since $\lim _{m \rightarrow \pm \infty}\left|a_{m}\right| r^{m}=0$ for any $r \in\left[r_{0}, r_{1}\right]$ by definition, there exists an integer $n$ such that $|f|_{r}=\left|a_{n}\right| r^{n}$.

Definition $2.1([3,2.3])$. Let $f=\sum_{-\infty<n<\infty} a_{n} x^{n}$ be a Laurent series which converges on $C\left[r_{0}, r_{1}\right]$. For $r \in\left[r_{0}, r_{1}\right]$, we define

$$
\begin{gathered}
K(r, f)=\max \left\{n \in Z ;|f|_{r}=\left|a_{n}\right| r^{n}\right\}, \\
k(r, f)=\min \left\{n \in \boldsymbol{Z} ;|f|_{r}=\left|a_{n}\right| r^{n}\right\} .
\end{gathered}
$$

In the special case where $r=0$ and $f(0)=0$, we define

$$
k(0, f)=0, \quad K(0, f)=\inf \left\{n ; a_{n} \neq 0\right\} .
$$

We call $r \in\left[r_{0}, r_{1}\right]$ a critical radius if $K(r, f)>k(r, f)$ and a regular radius if $K(r, f)=$ $k(r, f)$.

By [3, Proposition 2.3.2], we have $K(s, f g)=K(s, f)+K(s, g)$ and $k(s, f g)=$ $k(s, f)+k(s, g)$ for $0 \neq f, g \in \mathcal{A}[r, r]$. In particular, $K(s, 1 / f)=-K(s, f)$ and $k(s, 1 / f)=$ $-k(s, f)$. Let $f$ be a nonzero Laurent series which converges on $C\left[r_{0}, r_{1}\right]$. The set of critical radii of $f$ is finite [3, Proposition 2.3.1]. Let $r$ be a regular radius of $f$. For any $x \in \bar{K}$ such that $|x|=r$, we have $|f(x)|=|f|_{r}$. Indeed $\sup _{n}\left|a_{n} x^{n}\right|=\left|a_{k(r, f)}\right| r^{k(r, f)}$ and $\left|a_{n} x^{n}\right|<\left|a_{k(r, f)}\right| r^{k(r, f)}$ if $n \neq k(r, f)$. On the other hand, when $r$ is a critical radius, $f$ has $K(r, f)-k(r, f)$ zeros on $C[r, r]$ with counting multiplicities.

THEOREM 2.2 (Weierstrass preparation theorem [3, Proposition 2.4.3]). Let $f$ be an element of $\mathcal{A}\left[r_{1}, r_{2}\right]$. Let $r$ be a real number such that $r_{1} \leq r \leq r_{2}$. Let $d=K(r, f)-k(r, f)$. Then there exists a unique pair $(P, u)$ of a polynomial $P \in \bar{K}[x]$ of degree $d$ with $P(0)=$ $1, k(P, r)=0$ and $K(P, r)=d$, and an analytic function $u$ on $C\left[r_{1}, r_{2}\right]$ with $k(u, r)=$ $K(u, r)$ and $f=P u$.

We see by the Weierstrass preparation theorem that, for $f \in \mathcal{A}\left[r_{1}, r_{2}\right]$, the set $\{x \in$ $\left.C\left[r_{1}, r_{2}\right] ; f(x)=0\right\}$ is algebraic over $K$. When we regard $\log |f|_{r}$ as a function of $\log r$, we have the following proposition. 
Proposition 2.3. Let $f$ be an analytic function on $C\left[r_{0}, r_{1}\right]$. For $r \in\left[r_{0}, r_{1}\right]$, the map $\log r \longmapsto \log |f|_{r}$ is a piecewise linear continuous function. When $r$ is a regular radius, the slope of $\log |f|_{r}$ at $\log r$ is $K(r, f)$.

2.2. Valuation polygon. Let $f$ be an element of $\mathcal{A}[l, 1)$. In this subsection, we will define the valuation polygon of $f$. The valuation polygon of $f$ is determined by the Newton polygon of $f$. Conversely, the Newton polygon is determined by the valuation polygon of $f$. The exact order of log-growth of $f$ is determined by the valuation polygon of $f$ or the Newton polygon of $f$ (see the definition of the exact order of log-growth of $f$ in Definition 2.8). Therefore, if we want to know the exact order of log-growth of $f$, then we have only to know either the valuation polygon of $f$ or the Newton polygon of $f$.

Definition 2.4. For $f=\sum_{n=-\infty}^{\infty} a_{n} x^{n} \in \mathcal{A}[l, 1)$, the graph of the function

$$
t \mapsto \inf _{\infty>n>-\infty}\left(-\log \left|a_{n}\right|-n t\right)=-\log |f|_{e^{t}} \quad(\log l \leq t<0)
$$

is called the valuation polygon of Laurent series $f$.

The valuation polygon of $f$ is decided by the numbers of zeros of $f$ over $C\left[r_{n}, r_{n}\right]$ (equal to $\left.K\left(r_{n}, f\right)-k\left(r_{n}, f\right)\right)$ for the critical radii $r_{n}$ of $f$ and the initial condition $\left(K(l, f), a_{K(l, f)}\right)$. However, if we know the data of the critical radii $\left\{r_{n}\right\}$ of $f$ and the numbers of zeros of $f$ over $C\left[r_{n}, r_{n}\right]$, then we can compute the exact order of log-growth of $f$ (see Robert [15, p. 297-p. 304]).

Let $f \in \mathcal{A}[l, 1)$. By Proposition 2.3, there exist locally constant functions $A(r), B(r)$ such that $\log |f|_{r}=A(r) \log r+B(r)$. We obtain $A(r)=K(r, f)$ and $B(r)=\log \left|a_{K(r, f)}\right|$.

The function $-\log |f|_{e^{t}}$ is not differentiable at $t$ (i.e., $\left(t,-\log |f|_{e^{t}}\right)$ is a vertex of the valuation polygon of $f$ ) if and only if $t$ is a critical radius of $f$. If $t$ is a critical radius of $f$, then $K(t, f)-k(t, f)$ is equal to the difference of the slopes of $\log |f|_{e^{r}}$ at $r=t+\varepsilon$ and $r=t-\varepsilon$ for a sufficiently small real number $\varepsilon$. Therefore, if we know the number of zeros of $f$ over $C\left(r_{n}, r_{n}\right)$ (equal to $K\left(r_{n}, f\right)-k\left(r_{n}, f\right)$ ) for every critical radius $r_{n}$ of $f$ and the initial condition $\left(K(l, f), a_{K(l, f)}\right)$, then we can compute the valuation polygon of $f$ inductively.

Let $f, g$ be elements of $\mathcal{A}[l, 1)$. By definition, $|f|_{r}=|g|_{r}(l \leq r<1)$ if and only if the valuation polygon of $f$ coincides with that of $g$ on $[-\log l, 0)$. Assume that $g$ is nonconstant and has only finite zeros. The valuation polygon of $f$ does not coincide with any translation of the valuation polygon of $g f$ over $[-\log l, 0)$. However, there exists $r \in[l, 1)$ such that the set of critical radii of $f$ coincides with that of $f g$ over $[r, 1)$, and for each critical radius $s \in[r, 1)$ of $f$, we have $K(s, f)-k(s, f)=K(s, f g)-k(s, f g)$ (i.e., the valuation polygon of $f$ coincides with that of $g f$ over $[-\log r, 0)$ except for the initial condition). Indeed, by [3, Proposition 2.3.2], we have $K(s, f g)=K(s, f)+K(s, g)$ and $k(s, f g)=k(s, f)+k(s, g)$. If we choose $r \in[l, 1)$ such that $g$ has no zeros on $C[r, 1)$, then we have $K(s, f g)-k(s, f g)=K(s, f)-k(s, f)$ for $s \in[r, 1)$. Note that $s$ is a critical radius of $f$ if and only if $K(s, f)-k(s, f)>0$. Therefore, the set of critical radii of $f$ coincides with that of $f g$ over $[r, 1)$. 
We will explain the relation between the valuation polygon and the Newton polygon of $f=\sum_{n=0}^{\infty} a_{n} x^{n}$. Note that, when a real number $\rho$ is a critical radius, $\left(\log \rho, \inf _{n}\left\{-\log \left|a_{n}\right|-\right.\right.$ $n \log \rho\})$ is a vertex of the valuation polygon of $f$. Let $\left(n,-\log \left|a_{n}\right|\right)$ and $\left(m,-\log \left|a_{m}\right|\right)$ be adjacent verteces of the Newton polygon of $f$. The slope of the Newton polygon between these points is

$$
\log \rho=\frac{-\log \left|a_{n}\right|+\log \left|a_{m}\right|}{n-m} .
$$

Then, we have

$$
\log \rho^{n-m}=\log \frac{\left|a_{m}\right|}{\left|a_{n}\right|} .
$$

Therefore, $\rho$ is a critical radius since $\left|a_{n}\right| \rho^{n}=\left|a_{m}\right| \rho^{m}$. Conversely suppose $\rho$ is a critical radius. By the definition of critical radius, we have $k(\rho, f)<K(\rho, f)$ and

$$
\left|a_{K(\rho, f)}\right| \rho^{K(\rho, f)}=\left|a_{k(\rho, f)}\right| \rho^{k(\rho, f)} .
$$

Hence,

$$
\log \rho=-\frac{\log \left|a_{K(\rho, f)}\right|-\log \left|a_{k(\rho, f)}\right|}{K(\rho, f)-k(\rho, f)} .
$$

This concludes that $\log \rho$ is a slope of the Newton polygon of $f$ because $(k(\rho, f)$, $\left.-\log \left|a_{k(\rho, f)}\right|\right)$ and $\left(K(\rho, f),-\log \left|a_{K(\rho, f)}\right|\right)$ are adjacent verteces of the Newton polygon of $f$.

2.3. Poisson-Jensen formula. In this section, we review the Poisson-Jensen formula $[3,2.5]$. Let $f$ be an analytic function on $C\left[r_{1}, r_{2}\right)$ which is not identically zero. For $r \in$ $\left[r_{1}, r_{2}\right)$, we define a counting function $N(r, f)$ by

$$
N(r, f)=\sum_{\substack{0 \neq z \in C\left[r_{1}, r_{2}\right] \\ f(z)=0}} \log \frac{r}{|z|}+ \begin{cases}k\left(r_{1}, f\right) \log r+\log \left|a_{k\left(r_{1}, f\right)}\right| & r_{1}>0 \\ K(0, f) \log r & r_{1}=0 .\end{cases}
$$

For $r_{1}>0$ (resp. $r_{1}=0$ ), let $\left\{l_{n}\right\}$ be the strictly increasing sequence of the positive real numbers such that $\left\{l_{n}\right\}$ is the set of critical radii of $f$ on the interval $\left[r_{1}, r_{2}\right)$ (resp. $\left(r_{1}, r_{2}\right)$ ). For any real number $r$ such that $r_{1} \leq r<r_{2}$, take the greatest integer $k$ such that $l_{k} \leq r$. Then we have

$$
N(r, f)=K(r, f) \log r-\sum_{i=1}^{k}\left(K\left(l_{i}, f\right)-k\left(l_{i}, f\right)\right) \log l_{i} \quad \text { if } r_{1}=0,
$$

and

$$
N(r, f)=K(r, f) \log r+\log \left|a_{k\left(r_{1}, f\right)}\right|-\sum_{i=1}^{k}\left(K\left(l_{i}, f\right)-k\left(l_{i}, f\right)\right) \log l_{i} \quad \text { if } r_{1}>0 .
$$

If there is no integers $k$ with $l_{k} \leq r$, then $N(r, f)=K(r, f) \log r$. 
THEOREM 2.5 (Poisson-Jensen formula [3, Therem 2.5.1]). Let $f(z)=\sum_{n \in Z} a_{n} z^{n}$ be an analytic function on $C\left[r_{1}, r_{2}\right)$ with $r_{2} \leq \infty$. Then, for all $r \in\left[r_{1}, r_{2}\right)$, we have

$$
N(r, f)=\log |f|_{r} \quad \text { if } r_{1}>0,
$$

and

$$
N(r, f)+\log \left|a_{K(0, f)}\right|=\log |f|_{r} \quad \text { if } r_{1}=0 .
$$

2.4. The Robba ring. We define the Robba ring $\mathcal{R}$ over $K$ by

$$
\mathcal{R}=\left\{\begin{array}{cc}
\sum_{n=-\infty}^{\infty} a_{n} x^{n} ; & \begin{array}{c}
a_{n} \in K,\left|a_{n}\right| r^{n} \rightarrow 0(n \rightarrow \infty) \text { for all } 0<r<1 \\
\left|a_{n}\right| s^{n} \rightarrow 0(n \rightarrow-\infty) \text { for some } 0<s<1
\end{array}
\end{array}\right\} .
$$

We regard $\mathcal{A}[0,1)$ as a subring of $\mathcal{R}$. We define the bounded Robba ring $\mathcal{R}_{0}$ by

$$
\mathcal{R}_{0}=\left\{\sum_{n=-\infty}^{\infty} a_{n} x^{n} \in \mathcal{R} ; \sup _{n>0}\left|a_{n}\right|<\infty\right\} .
$$

Although $\mathcal{R}_{0}$ has the Gauss norm by definition, it is not complete under this norm. We regard $K[[x]]_{0}$ as a subring of $\mathcal{R}_{0} . \mathcal{R}$ and $\mathcal{R}_{0}$ do not have the norm $\mid{ }_{r}$ for $0<r<1$. However, for each element $f$ of $\mathcal{R}$ (resp. $\mathcal{R}_{0}$ ), there exists a real number $r_{0}$ such that $\mathcal{A}\left[r_{0}, 1\right)$ contains $f$. Then we can define $|f|_{r}$ for $r_{0} \leq r<1$ (resp. $r_{0} \leq r \leq 1$ ). $\mathcal{R}$ and $\mathcal{R}_{0}$ have Frobenius endomorphisms $\phi: \sum a_{n} x^{n} \mapsto \sum a_{n} x^{p n}$.

Since $K$ is a discrete valued field, we obtain the following lemma.

LEMMA 2.6 ([7], [11], [12] and [16]). Let $f=\sum_{n=-\infty}^{\infty} a_{n} x^{n}$ be an element of $\mathcal{R}_{0}$. Then, there exists a real number $r_{0}$ such that, for all $r \in\left[r_{0}, 1\right)$, there exist $t \in \boldsymbol{Q}$ and $s \in \boldsymbol{Z}$ with $|f|_{r}=r^{s} p^{t}$.

Proof. We assume that $f=\sum a_{n} x^{n} \in \mathcal{R}_{0}$ is analytic on $C[l, 1)$. We have only to prove that $f$ has only finite zeros on $C[l, 1)$. Indeed we can choose $r_{0} \in[l, 1)$ such that $f$ has no zeros on $C\left[r_{0}, 1\right)$, and then $s=K\left(r_{0}, f\right)$ and $t=\log _{p}\left|a_{K\left(r_{0}, f\right)}\right|$. Suppose that $f$ has infinite zeros on $C[l, 1)$. Since $f$ has only finite zeros on each critical radius, $f$ has infinite critical radii on $[l, 1)$. We take strictly increasing sequence $\left\{r_{n}\right\}_{n \in N}$ of critical radii of $f$ on the interval $[l, 1)$. By the definition of critical radius, we have $k\left(r_{i}, f\right)<K\left(r_{i}, f\right)$ and

$$
\left|a_{k\left(r_{i}, f\right)}\right| r_{i}^{k\left(r_{i}, f\right)}=\left|a_{K\left(r_{i}, f\right)}\right| r_{i}^{K\left(r_{i}, f\right)} \quad(i=1,2, \ldots) .
$$

Since $K\left(r_{i}, f\right)=k\left(r_{i+1}, f\right)$ by definition and $r_{i}<1$ for all $i \in N$, we have

$$
\left|a_{k\left(r_{1}, f\right)}\right|<\left|a_{k\left(r_{2}, f\right)}\right|<\cdots<\left|a_{k\left(r_{m}, f\right)}\right|<\cdots .
$$

Since $K$ is a discretely valued field, $f$ is not bounded. This contradicts the assumption that $f \in \mathcal{R}_{0}$.

By the above lemma, we see $\mathcal{R}_{0}$ is a field. In fact, $\mathcal{R}_{0}$ is a henselian field [7], [11, Lemma 15.1.3], [12, Proposition 3.2] and [16]. 
REMARK 2.7. Assume that $K$ has a lift $\sigma$ of Frobenius endomorphism on the residue field of $K$. Then $\sigma$ is extended to $\mathcal{R}_{0}$ so that $\left|\sigma(x)-x^{p}\right|_{1}<1$. Then $\sigma$ can be extended to $\mathcal{R}$ naturally. Even if we replace $\phi$ by $\sigma$, we can establish Theorems 2.10 and 4.1 because, for any $f \in \mathcal{R}$, there exists a positive real number $r_{0}<1$ such that for all $r \in\left[r_{0}, 1\right)$, $\left|f^{\phi}\right|_{r}=\left|f^{\sigma}\right|_{r}=|f|_{r^{p}}$ (see [11, 15.2.4]).

\subsection{Log-growth.}

Definition 2.8. Let $f=\sum_{n=-\infty}^{\infty} a_{n} x^{n}$ be an element of the Robba ring $\mathcal{R}$ and let $\alpha$ be a nonnegative real number. We say $f$ is of logarithmic growth (or log-growth) $\alpha$ if

$$
\limsup _{n \rightarrow \infty} \frac{\left|a_{n}\right|}{n^{\alpha}}<\infty \text {. }
$$

If moreover, $f$ is not of $\log$-growth $\beta$ for any real number $\beta$ such that $0 \leq \beta<\alpha$, then we say $f$ is exactly of $\log$-growth $\alpha$. When $f$ is exactly of $\log$-growth $\alpha, \alpha$ is said to be the exact order of log-growth of $f$.

The following result is given in [6, Proposition 2.3.3].

Proposition 2.9. Let $r_{0}$ be a real number such that $0 \leq r_{0}<1$. For $f \in \mathcal{A}\left[r_{0}, 1\right)$, $f$ is of log-growth $\alpha$ if and only if for $r_{0}<r<1$,

$$
|f|_{r}=O\left(\log ^{-\alpha}(1 / r)\right) .
$$

When $f \in \mathcal{R}$ satisfies a Frobenius equation (1), the order of log-growth of $f$ is given as follows (see [4, Proposition 6.12] and [11, Theorem18.4.5]).

THEOREM 2.10. Let $f$ be a nonzero element of the Robba ring $\mathcal{R}$. Suppose $f$ satisfies a Frobenius equation

$$
a_{0} f+a_{1} f^{\phi}+\cdots+a_{n} f^{\phi^{n}}=0\left(a_{i} \in \mathcal{R}_{0}, a_{0} \neq 0\right) .
$$

If $t$ is the first slope of the Newton polygon of the polynomial

$$
a_{0}+a_{1} X+\cdots+a_{n} X^{n}
$$

with respect to the Gauss norm, then $f$ is of log-growth $-t$.

PROOF. Note that, for $g \in \mathcal{A}\left[r_{0}, 1\right)$ and $r \in\left[r_{0}, 1\right)$, we have $\left|g^{\phi}\right|_{r}=|g|_{r}$. We may assume $a_{0}=-1$. Then

$$
\begin{aligned}
f & =a_{1} f^{\phi}+\cdots+a_{n} f^{\phi^{n}} \\
& =a_{1} \phi\left(a_{1} f^{\phi}+\cdots+a_{n} f^{\phi^{n}}\right)+a_{2} f^{\phi^{2}}+\cdots+a_{n} f^{\phi^{n}} \\
& =\left(a_{1} a_{1}^{\phi}+a_{2}\right) f^{\phi^{2}}+\cdots+\left(a_{n}+a_{1} a_{n-1}^{\phi}\right) f^{\phi^{n}}+a_{1} a_{n}^{\phi} f^{\phi^{n+1}} .
\end{aligned}
$$

Thus $f$ satisfies the equality

$$
f=A_{m}^{1} f^{\phi^{m+1}}+\cdots+A_{m}^{n} f^{\phi^{m+n}}\left(A_{m}^{i} \in \mathcal{R}_{0}\right),
$$

where $A_{m}^{i}$ 's satisfy the relations

$$
A_{0}^{i}=a_{i} \quad(1 \leq i \leq n),
$$




$$
\begin{aligned}
& A_{m+1}^{i}=A_{m}^{i+1}+A_{m}^{1} a_{i}^{\phi^{m+1}}(1 \leq i \leq n-1), \\
& A_{m+1}^{n}=A_{m}^{1} a_{n}^{\phi^{m+1}} .
\end{aligned}
$$

By Lemma 2.6, there exist a real number $r_{0}$, integers $s_{i}$ and rational numbers $t_{i}$ for $i=$ $1,2, \ldots, n$ such that $f, f^{\phi}, \ldots, f^{\phi^{n}} \in \mathcal{A}\left[r_{0}, 1\right)$ and

$$
a_{i} \in \mathcal{A}\left[r_{0}, 1\right) \text { with }\left|a_{i}\right|_{r}=r^{s_{i}} p^{t_{i}} \text { for } r \in\left[r_{0}, 1\right) .
$$

By induction, we can easily see $A_{m}^{i} \in \mathcal{A}\left[r_{0}^{1 / p^{m}}, 1\right)$ for all $i$ because if $g \in \mathcal{A}\left[r_{0}, 1\right)$ then $g^{\phi} \in \mathcal{A}\left[r_{0}^{1 / p}, 1\right)$ in general. Let $l$ be an integer such that $t_{l} / l=\max _{i}\left\{t_{i} / i\right\}$. Then, $t_{l} / l$ is equal to $-t$ by definition. We have to prove that $f$ is of $\log$-growth $t_{l} / l$.

LEMMA 2.11. Let $s=\max \left|s_{i}\right|_{\infty}\left(||_{\infty}\right.$ is the usual absolute value). For all $r \in\left[r_{0}, 1\right)$,

$$
\left|A_{m}^{i}\right|_{r^{1 / p^{m}}} \leq r^{-s\left(1+1 / p+\cdots+1 / p^{m}\right)} p^{(m+i) t_{l} / l}(i=0, \ldots, n) .
$$

Proof. We use induction on $m$. For $m=0$ and $r \in\left[r_{0}, 1\right)$, we have

$$
\left|A_{0}^{i}\right|_{r}=\left|a_{i}\right|_{r}=r^{s_{i}} p^{t_{i}} \leq r^{-s} p^{i t_{i} / i} \leq r^{-s} p^{i t_{l} / l} .
$$

Therefore, the case $m=0$ is true.

We assume that the assertion is true for $m=k$. For $1 \leq i \leq n-1$ and $r \in\left[r_{0}, 1\right)$, we have

$$
\left|A_{k+1}^{i}\right|_{r^{1 / p^{k+1}}} \leq \max \left(\left|A_{k}^{i+1}\right|_{r^{1 / p^{k+1}}},\left|a_{i}^{\phi^{k+1}} A_{k}^{1}\right|_{r^{1 / p^{k+1}}}\right) .
$$

We evaluate the right-hand side. We set $u=r^{1 / p} \in\left[r_{0}, 1\right)$. Then by the induction hypothesis, we have

$$
\begin{aligned}
\left|A_{k}^{i+1}\right|_{r^{1 / p^{k+1}}}=\left|A_{k}^{i+1}\right|_{u^{1 / p^{k}}} & \leq u^{-s\left(1+\cdots+1 / p^{k}\right)} p^{(k+i+1) t_{l} / l} \\
& <r^{-s\left(1+\cdots+1 / p^{k+1}\right)} p^{(k+i+1) t_{l} / l} \\
\left|a_{i}^{\phi^{k+1}} A_{k}^{1}\right|_{r^{1 / p^{k+1}}} & =\left|a_{i}\right|_{r}\left|A_{k}^{1}\right|_{r^{1 / p^{k+1}}} \\
& \leq r^{-s} p^{i t_{l} / l} \times u^{-s\left(1+\cdots+1 / p^{k}\right)} p^{(k+1) t_{l} / l} \\
& =r^{-s\left(1+1 / p+\cdots+1 / p^{k+1}\right)} p^{(k+i+1) t_{l} / l} .
\end{aligned}
$$

Therefore, the assertion is true for $i=1, \ldots, n-1$. We can also prove the assertion for $i=n$ by the same way. Hence the lemma is proved.

We continue the proof of Theorem 2.10. We fix $r_{1} \in\left[r_{0}, 1\right)$. Note $r_{1}^{-s\left(1+\cdots+1 / p^{m}\right)}<$ $r_{1}^{-s p /(p-1)}$ for all $m$. By Lemma 2.11,

$$
\begin{aligned}
|f|_{r_{1} 1 / p^{m}} & \leq \max _{i}\left(\left|A_{m}^{i}\right|_{r_{1} 1 / p^{m}}\left|f^{\phi^{i}}\right|_{r_{1}}\right) \\
& =O\left(p^{m t_{l} / l}\right)=O\left(\log ^{-t_{l} / l}\left(1 / r_{1}^{1 / p^{m}}\right)\right)(m \rightarrow \infty) .
\end{aligned}
$$

We may assume that $|f|_{v}$ is an increasing function over $\left[r_{0}, 1\right)$. For any $v \in\left[r_{0}, 1\right)$, there exists an integer $m \in N$ such that $r_{1}^{1 / p^{m}} \leq v<r_{1}^{1 / p^{m+1}}$. Since $|f|_{r_{1} 1 / p^{m}} \leq|f|_{\nu} \leq|f|_{r_{1} 1 / p^{m+1}}$, 
we have

$$
|f|_{v}=O\left(\log ^{-t_{l} / l}(1 / v)\right)(v \rightarrow 1)
$$

by the inequality above. Therefore, $f$ is of log-growth $t_{l} / l$ by Proposition 2.9.

REMARK 2.12. Under the assumption of Theorem $2.10, f$ may not be exactly of loggrowth $-t$. Let $f$ be a nonzero element of the Robba $\operatorname{ring} \mathcal{R}$ which satisfies (3). Then $f$ also satisfies a Frobenius equation of degree $n+1$ :

$$
\frac{1}{p^{k}} \phi\left(a_{0} f+a_{1} f^{\phi}+\cdots+a_{n} f^{\phi^{n}}\right)+\left(a_{0} f+a_{1} f^{\phi}+\cdots+a_{n} f^{\phi^{n}}\right)=0 .
$$

If we take an integer $k$ large enough, the first slope of the Newton polygon corresponding (5) is larger than $\inf \{\alpha ; f$ is of log-growth $\alpha\}$.

PROPOSITION 2.13. Let $f$ be an element in $\mathcal{R}$. If there exists a real number $r_{0} \in[0,1)$ such that $-K(r, f) \log r \leq \alpha$ for any $r \in\left[r_{0}, 1\right)$, then $f$ is of log-growth $\alpha$.

PROOF. According to [10, p.21], we have

$$
N(r, f)=\int_{r_{0}}^{r} \frac{K(t, f)}{t} d t+O(1) \quad\left(r \in\left[r_{0}, 1\right)\right) .
$$

By this equality, we have

$$
\begin{aligned}
N(r, f) & =\int_{r_{0}}^{r} \frac{K(t, f)}{t} d t+O(1) \\
& =\int_{r_{0}}^{r} \frac{-K(t, f) \log t}{-t \log t} d t+O(1) \\
& \leq \int_{r_{0}}^{r} \frac{\alpha}{-t \log t} d t+O(1) \\
& =-\alpha \log (-\log r)+O(1)
\end{aligned}
$$

Theorem 2.5 implies the inequality

$$
\log |f|_{r} \leq-\alpha \log (-\log r)+O(1) .
$$

We see that $f$ is of log-growth $\alpha$.

\section{Critical radius.}

3.1. Condition $\left(B_{i}\right)$. Let $K$ be a complete discrete valued field with mixed characteristic $(0, p)$ and $\phi$ a Frobenius endomorphism $\sum a_{n} x^{n} \mapsto \sum a_{n} x^{p n}$. Let $f=\sum a_{n} x^{n} \in$ $\mathcal{A}\left[r_{0}, 1\right)$. We say $\alpha \in \bar{K}$ is the zero of $f$ if $f(\alpha)=0$, and we count the number of zeros of $f$ with multiplicities of zeros. Let $\alpha$ be an element of the set $\{\alpha \in \bar{K} ;|\alpha| \in$ $\left[r_{0}, 1\right)$ and $\left.f(\alpha)=0\right\}$ and $\alpha^{1 / p}$ a fixed primitive $p$-th root of $\alpha$. Then $\alpha^{1 / p} \zeta^{i}$ is an element of the set $\left\{a \in \bar{K} ; a \in\left[r_{0}^{1 / p}, 1\right), f^{\phi}(a)=0\right\}$ for all $i=0,1, \ldots, p-1$, where $\zeta$ is a primitive $p$-th root of unity. Conversely, any zero of $f^{\phi}$ over $C\left[r_{0}^{1 / p}, 1\right)$ can be written like this. We can easily see the following proposition holds. 
PROPOSITION 3.1. Let $f$ be an element of $\mathcal{A}\left[r_{0}, 1\right)$, and $\left\{r_{i}\right\}_{i}$ the set of critical radii of $f$ over $[r, 1)$. Then the set of critical radii of $f^{\phi}$ over $\left[r_{0}^{1 / p}, 1\right)$ is $\left\{r_{i}^{1 / p}\right\}$. Moreover, we have $p k(l, f)=k\left(l^{1 / p}, f^{\phi}\right)$ and $p K(l, f)=K\left(l^{1 / p}, f^{\phi}\right)$ for any $l \in\left[r_{0}, 1\right)$.

PROPOSITION 3.2. Let $f$ be an element of $\mathcal{A}[l, 1)$. Let $\left\{r_{n}\right\}_{n \geq 1}$ be the strictly increasing sequence such that $\left\{r_{n}\right\}$ is the set of critical radii of $f$ on the interval $[l, 1)($ resp. $(0,1))$ if $l>0$ (resp. $l=0)$. For $r \in[l, 1)$ (resp. $r \in(0,1)$ ), let $n$ and $m$ be the greatest integers with $r_{n} \leq r$ and $r_{m}^{1 / p} \leq r$. Then we have an equality

$$
\begin{aligned}
\log |f|_{r}-\log \left|f^{\phi^{i}}\right|_{r}= & \left(K\left(r_{n}, f\right)-k\left(r_{m}^{1 / p^{i}}, f^{\phi^{i}}\right)\right) \log r \\
& -\sum_{j=m+1}^{n}\left(K\left(r_{j}, f\right)-k\left(r_{j}, f\right)\right) \log r_{j} .
\end{aligned}
$$

Proof. By using Proposition 3.1 and the Poisson-Jensen formula, we obtain

$$
\begin{aligned}
\log |f|_{r}-\log \left|f^{\phi^{i}}\right|_{r}= & N(r, f)-N\left(r, f^{\phi^{i}}\right) \\
= & \left(K\left(r_{n}, f\right)-k\left(r_{m}^{1 / p^{i}}, f^{\phi^{i}}\right)\right) \log r \\
& -\sum_{j=1}^{n}\left(K\left(r_{j}, f\right)-k\left(r_{j}, f\right)\right) \log r_{j} \\
& +\sum_{j=1}^{m}\left(K\left(r_{i}^{1 / p^{i}}, f^{\phi^{i}}\right)-k\left(r_{j}^{1 / p^{i}}, f^{\phi^{i}}\right)\right) \log r_{j}^{1 / p^{i}} \\
= & \left(K\left(r_{n}, f\right)-k\left(r_{m}^{1 / p^{i}}, f^{\phi^{i}}\right)\right) \log r \\
& -\sum_{j=m+1}^{n}\left(K\left(r_{j}, f\right)-k\left(r_{j}, f\right)\right) \log r_{j} .
\end{aligned}
$$

Let $f$ be a nonzero element of the Robba ring $\mathcal{R}$ which satisfies a Frobenius equation

$$
a_{0} f+a_{1} f^{\phi}+\cdots+a_{n} f^{\phi^{n}}=0 \quad\left(a_{i} \in \mathcal{R}_{0}, a_{0} \neq 0\right) .
$$

Let $i=\min _{1 \leq j \leq n}\left\{j ; a_{j} \neq 0\right\}$. If $|f|_{r}$ is an increasing function with respect to $r$, then we have

$$
\begin{aligned}
\log |f|_{r}-\log \left|f^{\phi^{i}}\right|_{r} & =\log \left|a_{i} f^{\phi^{i}}+\cdots+a_{n} f^{\phi^{n}}\right|_{r}-\log \left|a_{i}\right|_{r}-\log \left|f^{\phi^{i}}\right|_{r} \\
& \leq \max _{j \geq i}\left\{\log \left|a_{j} f^{\phi^{j}}\right|_{r}\right\}-\log \left|a_{i}\right|_{r}-\log \left|f^{\phi^{i}}\right|_{r} \\
& =\max _{j \geq i}\left\{\log \left|f^{\phi^{j}}\right|_{r}-\log \left|f^{\phi^{i}}\right|_{r}+\log \left|a_{j}\right|_{r}\right\}-\log \left|a_{i}\right|_{r} \\
& \leq \max _{j \geq i}\left\{\log \left|a_{j}\right|\right\}-\log \left|a_{i}\right|_{r}+O(1)=O(1)
\end{aligned}
$$


DEFINITION 3.3. Let $i$ be a natural number. For a nonzero element $f \in \mathcal{R}$, we say $f$ satisfies the condition $\left(B_{i}\right)$ if there exists a real number $r_{0} \in[0,1)$ such that

$$
\log |f|_{r}-\log \left|f^{\phi^{i}}\right|_{r}=O(1) \text { for } r \in\left[r_{0}, 1\right) .
$$

The following lemma can be seen easily.

LEMMA 3.4. Let $f, g$ be nonzero elements of the Robba ring $\mathcal{R}$.

(i) If $f$ satisfies a Frobenius equation (7), then $f$ satisfies $\left(B_{i}\right)$ for some $i$.

(ii) If $f$ satisfies $\left(B_{i}\right)$, then for any $k \in N, f$ satisfies $\left(B_{k i}\right)$.

(iii) $f$ satisfies $\left(B_{i}\right)$ if and only if $f^{\phi}$ satisfies $\left(B_{i}\right)$.

(iv) If $f, g$ satisfy $\left(B_{i}\right)$, then $f g$ satisfies $\left(B_{i}\right)$.

By the Poisson-Jensen formula, we obtain the following proposition.

PROPOSITION 3.5. Let $f$ be a nonzero element of $\mathcal{A}\left[r_{0}^{p^{i}}, 1\right)$ which satisfies $\left(B_{i}\right)$. If we write for $r \in\left[r_{0}, 1\right)$

$$
\log |f|_{r}-\log \left|f^{\phi^{i}}\right|_{r}=A(r) \log r+B(r),
$$

where $A(r), B(r)$ are locally constant functions, defined by Proposition 2.3, then we have

$$
\begin{gathered}
|A(r) \log r|_{\infty} \text { is bounded on }\left(r_{0}, 1\right), \\
0 \leq B(r) \text { is bounded on }\left(r_{0}, 1\right), \\
B(r) \in \log |K| .
\end{gathered}
$$

In particular, the set $\left\{B(r) ; r \in\left[r_{0}, 1\right)\right\}$ is finite.

PROOF. We will prove the assertion in the case where $i=1$. The other cases are proved similarly.

The assertion (10) follows from the definition of the norm ||$_{r}$. It follows from (6) that $0 \leq B(r)$. Since $f$ satisfies $\left(B_{1}\right)$, the assertion (8) implies the assertion (9). Therefore, we have only to prove the assertion (8). According to [10, p.21],

$$
N(r, f)=\int_{r_{0}}^{r} \frac{K(t, f)}{t} d t+O(1) \quad\left(r \in\left[r_{0}, 1\right)\right)
$$

where $O(1)$ is a bounded function. Therefore, for any $r \in\left[r_{0}^{1 / p}, 1\right)$, we have

$$
\begin{aligned}
\log |f|_{r}-\log \left|f^{\phi}\right|_{r} & =\log |f|_{r}-\log |f|_{r^{p}} \\
& =N(r, f)-N\left(r^{p}, f\right) \\
& =\int_{r^{p}}^{r} \frac{K(t, f)}{t} d t+O(1) \\
& \geq \int_{r^{p}}^{r} \frac{K\left(r^{p}, f\right)}{t} d t+O(1) \\
& =-\frac{p-1}{p} K\left(r^{p}, f\right) \log r^{p}+O(1) .
\end{aligned}
$$


Since $f$ satisfies $\left(B_{1}\right)$, the left-hand side is bounded for any $r \in\left[r_{0}, 1\right)$. Therefore, $|K(r, f) \log r|_{\infty}<\infty$. Since $A(r)=K(r, f)-K\left(r, f^{\phi}\right)$ by $(6)$, we have

$$
\begin{aligned}
|A(r) \log r|_{\infty} & \leq|K(r, f) \log r|_{\infty}+\left|K\left(r, f^{\phi}\right) \log r\right|_{\infty} \\
& =O(1)+\left|K\left(r^{p}, f\right) \log r^{p}\right|_{\infty}=O(1) .
\end{aligned}
$$

3.2. Condition $\left(A_{i}\right)$. Let $f$ be a nonzero element of the Robba ring $\mathcal{R}$ which satisfies $\left(B_{i}\right)$. By the definition of $\left(B_{i}\right)$, there exists a real number $r_{0}$ such that, for $r \in\left[r_{0}, 1\right)$,

$$
\log |f|_{r}-\log \left|f^{\phi^{i}}\right|_{r}=O(1) .
$$

However, we do not know the existence of $b \in \mathcal{R}_{0}$ and $r_{1} \in[0,1)$ such that

$$
\log |f|_{r}-\log \left|f^{\phi^{i}}\right|_{r}=\log |b|_{r} \quad \text { for } r \in\left[r_{1}, 1\right) .
$$

DEFINITION 3.6. Let $i$ be a natural number. For nonzero $f \in \mathcal{R}$, we say $f$ satisfies the condition $\left(A_{i}\right)$ if there exist $b \in \mathcal{R}_{0}$ and $l_{1} \in(0,1)$ such that

$$
\log |f|_{r}-\log \left|f^{\phi^{i}}\right|_{r}=\log |b|_{r} \text { for } r \in\left[l_{1}, 1\right) .
$$

The following lemma is verified easily.

LEMMA 3.7. Let $f$ be a nonzero element of the Robba ring $\mathcal{R}$.

(i) If $f$ satisfies $\left(A_{i}\right)$, then $f$ satisfies $\left(B_{i}\right)$ and $\left(A_{k i}\right)$ for any natural number $k$.

(ii) $f$ satisfies $\left(A_{i}\right)$ if and only if $f^{\phi}$ satisfies $\left(A_{i}\right)$.

(iii) If $f, g$ satisfy $\left(A_{i}\right)$, then $f g$ satisfies $\left(A_{i}\right)$.

When $f$ satisfies $\left(A_{i}\right)$, the exact order of log-growth of $f$ is calculated as follows.

PROPOSITION 3.8. Let $f$ be a nonzero element of the Robba ring $\mathcal{R}$ which satisfies $\left(A_{i}\right)$. Then $f$ is exactly of log-growth $\lim _{r \rightarrow 1^{-}}\left(\log _{p}|f|_{r}-\log _{p}\left|f^{\phi^{i}}\right|_{r}\right) / i$.

Proof. By definition, there exist an element $a \in \mathcal{R}_{0}$ and a real number $r_{0} \in(0,1)$ such that for all $r \in\left[r_{0}, 1\right)$

$$
\log |f|_{r}-\log \left|f^{\phi^{i}}\right|_{r}=\log |a|_{r} .
$$

By Lemma 2.6, there exist a real number $r_{1}$, an integer $k$ and a rational number $t$ such that $|a|_{r}=r^{k} p^{t}$ for any $r \in\left[r_{1}, 1\right)$. We will prove $f$ is exactly of log-growth $t / i$. We fix a real number $r$ such that $r \in\left[\max \left\{r_{0}, r_{1}\right\}, 1\right)$. Then

$$
|f|_{r^{1 / p^{l i}}}=|a|_{r^{1 / p^{l i}}}\left|f^{\phi^{i}}\right|_{r^{1 / p^{l i}}}=p^{t} r^{k / p^{l i}}\left|f^{\phi^{i}}\right|_{r^{1 / p^{l i}}}=p^{t} r^{k / p^{l i}}|f|_{r^{1 / p^{(l-1) i}}}
$$

for each integer $l$. Hence for any nonnegative integer $n$, we have

$$
|f|_{r^{1 / p^{n i}}}=p^{n t} r^{k\left(1 / p^{i}+1 / p^{2 i}+\cdots+1 / p^{n i}\right)}|f|_{r} .
$$

Since

$$
r^{k\left(1 / p^{i}+1 / p^{2 i}+\cdots+1 / p^{n i}\right)} \rightarrow r^{k /\left(p^{i}-1\right)}>0 \quad(n \rightarrow \infty),
$$


we have

$$
\begin{aligned}
|f|_{r^{1 / p^{n i}}}=O\left(p^{t n}\right)=O\left(\log ^{-t / i}\left(1 / r^{1 / p^{i n}}\right)\right) \quad(n \rightarrow \infty), \\
|f|_{r^{1 / p^{n i}}} \neq O\left(\log ^{-t / i}\left(1 / r^{1 / p^{n i}}\right)\right) \quad(n \rightarrow \infty) .
\end{aligned}
$$

By Proposition 2.9, $f$ is exactly of log-growth $t / i$.

The condition $\left(A_{i}\right)$ has the following properties.

Proposition 3.9. Let $f$ be a nonzero element of the Robba ring $\mathcal{R}$. Assume $f, f^{\phi^{i}} \in$ $\mathcal{A}\left[l_{1}, 1\right)$. Let $\left\{r_{n}\right\}_{n \in N}$ be the strictly increasing sequence of critical radii of $f$ over the interval $\left[l_{1}, 1\right)$. Then the following conditions are equivalent.

(i) $f$ satisfies $\left(A_{i}\right)$.

(ii) (a) There exist an $r \in\left[l_{1}, 1\right)$ and an integer $m$ such that $r_{k+m}=r_{k}^{1 / p^{i}}$ for any critical radius $r_{k}>r$, and

(b) for almost all critical radii $r_{k}$, the number of zeros of $f$ is equal to the number of zeros of $f^{\phi^{i}}$ over $C\left[r_{k}, r_{k}\right]$.

(iii) For any $r$ close enough to 1 , the difference between the number of zeros of $f$ and the number of zeros of $f^{\phi^{i}}$ over $C[r, 1)$ is constant.

(iv) For any $r \in\left[l_{1}, 1\right)$, the difference between the number of zeros of $f$ and the number of zeros of $f^{\phi^{i}}$ over $C[r, 1)$ is bounded.

Proof. (i) $\Rightarrow$ (ii). Assume $f$ satisfies (12) for some $b \in R_{0}$. Then for some $l_{1}^{\prime} \in\left[l_{1}, 1\right)$, the valuation polygon of $f$ coincides with that of $b f^{\phi^{i}}$ over $\left[l_{1}^{\prime}, 1\right)$. Therefore, the set of critical radii of $f$ coincides with that of $b f^{\phi^{i}}$. Hence, the number of zeros of $f$ over $C\left[r_{k}, r_{k}\right]$ coincides with that of $b f^{\phi^{i}}$ over $C\left[r_{k}, r_{k}\right]$. Since $b$ has only finite zeros over $C\left[l_{1}^{\prime}, 1\right)$ by Lemma 2.6, there exists a real number $l_{2}$ such that the set of critical radii of $f$ coincides with that of $f^{\phi^{i}}$ over $\left[l_{2}, 1\right)$ and, if $r_{k}>l_{2}$, the number of zeros of $f$ over $C\left[r_{k}, r_{k}\right]$ coincides with that of $f^{\phi^{i}}$ over $C\left[r_{k}, r_{k}\right]$. Hence the assertion (b) is true. If $r_{k}>l_{2}$, then there exists an integer $t$ such that $r_{k}=r_{t}^{1 / p^{i}}$ by Proposition 3.1 because the set of critical radii of $f$ coincides with that of $f^{\phi^{i}}$ over $\left[l_{2}, 1\right)$. Then we see $r_{k+n}=r_{t+n}^{1 / p^{i}}$ for any nonnegative integer $n$ and the assertion (ii) is true.

(ii) $\Rightarrow$ (iii). $\quad$ It is trivial because the zeros of $f$ exist only on the critical radius of $f$.

(iii) $\Rightarrow$ (i). By assumption, there exist an integer $A$ and real numbers $B$ and $l$ such that $\log |f|_{r}-\log \left|f^{\phi^{i}}\right|_{r}=A \log r+B$ for any $r \in[l, 1)$. Then it is obvious that $B \in \log |K|$. Therefore, there exists a rational function $b \in K(x)$ such that $\log |b|_{r}=A \log r+B$ for any $r \in[l, 1)$. Therefore, $f$ satisfies $\left(A_{i}\right)$.

Since (iii) $\Rightarrow$ (iv) is obvious, the rest is to prove (iv) $\Rightarrow$ (i). Write

$$
\log |f|_{r}-\log \left|f^{\phi^{i}}\right|_{r}=A(r) \log r+B(r),
$$

where $A, B$ are locally constant functions. Since $A(r)$ is bounded by assumption, $B(r)$ is also bounded. Moreover, we have $A(r) \in \boldsymbol{Z}$ and $B(r) \in \log |K|$. Therefore, the set $\{(A(r), B(r)) ; r \in[l, 1)\}$ is finite. Hence $\log |f|_{r}-\log \left|f^{\phi^{i}}\right|_{r}$ as a function of $\log r$ has 
only finite non-differentiable points. Therefore, for any $r$ close enough to $1, A(r)$ and $B(r)$ are constant. Hence (i) holds.

The next proposition follows from Propositions 3.8 and 3.9.

PROPOSITION 3.10. Let $f$ be a nonzero element of the Robba ring $\mathcal{R}$ which satisfies $\left(A_{i}\right)$. If we write $\left(\log _{p}|f|_{r}-\log _{p}\left|f^{\phi^{i}}\right|_{r}\right) / i$ as $(6)$, then $-\left(\sum_{j=m+1}^{n}\left(K\left(r_{j}, f\right)-\right.\right.$ $\left.\left.k\left(r_{j}, f\right)\right) \log _{p} r_{j}\right) / i$ is independent of $r$ for any $r$ close enough to 1 and it coincides with the exact order of log-growth of $f$.

EXAMPLE 3.11. (i) $v:=\log (1+x)=\sum_{n \geq 1}(-1)^{n-1} x^{n} / n$ has $N_{i}=p^{i}(p-1)$ zeros on the annulus $\left\{x \in Q_{p}^{\text {alg }} ;|x|=p^{-1 / p^{i}(p-1)}\right\}$ of radius $r_{i}=p^{-1 / p^{i}(p-1)}$ for $i \in\{0\} \cup$ $\boldsymbol{N}$ and one zero at the origin. By Proposition 3.1, $v$ satisfies the condition (ii) of Proposition 3.8 and hence $v$ satisfies $\left(A_{1}\right)$. For $r \in\left[p^{-1 /(p-1)}, 1\right)$, take the greatest integer $n$ such that $p^{-1 / p^{n}(p-1)}<r$. Then

$$
\begin{aligned}
\log _{p}|v|_{r}-\log _{p}\left|v^{\phi}\right|_{r}= & \left(K(r, v)-K\left(r, v^{\phi}\right)\right) \log _{p} r-\sum_{i=0}^{n} N_{i} \log _{p} r_{i}+\sum_{i=0}^{n-1} p N_{i} \log _{p} r_{i}^{1 / p} \\
= & \left(1+\sum_{i=0}^{n} N_{i}-p-\sum_{i=0}^{n-1} p N_{i}\right) \log _{p} r \\
& -\sum_{i=0}^{n} p^{i}(p-1) \log _{p} p^{-1 / p^{i}(p-1)} \\
& +\sum_{i=0}^{n-1} p^{i+1}(p-1) \log _{p} p^{-1 / p^{i+1}(p-1)} \\
= & 1 .
\end{aligned}
$$

$v$ is exactly of log-growth 1 by Proposition 3.8. On the other hand, $v$ satisfies a Frobenius equation. In fact, we see that $c:=v-v^{\phi} / p$ is an element of $\boldsymbol{Z}_{p}[[x]]$ by an easy calculation. Therefore, $v$ satisfies the Frobenius equation

$$
y-\left(\frac{1}{p}+\frac{c}{c^{\phi}}\right) y^{\phi}+\frac{c}{p c^{\phi}} y^{\phi^{2}}=0 .
$$

Needless to say, it is obvious that $v$ is exactly of log-growth 1 by definition.

(ii) See Tsuzuki [16, Example 6.26]. Assume $p \neq 2$. We set

$$
u=1+\sum_{n=1}^{\infty} \frac{((2 n-1) ! !)^{2}}{(8 \pi)^{n} n !} y^{n}
$$

where $\pi$ is a solution of equation $x^{p-1}=-p$ and $(2 n-1) ! !=1 \times 3 \times \cdots \times(2 n-1) . u$ satisfies the differential equation $4 y \delta_{y}^{2}(u)+4(y-2 \pi) \delta_{y}(u)+y u=0$ with $\delta_{y}=y d / d y$ and a Frobenius equation. By direct calculation, we can see the vertices of the Newton polygon of $u$ are $\left(\left(p^{i}-1\right) / 2,-i / 2\right)$ for $i \in\{0\} \cup N$, the critical radii of $u$ are $r_{i}=p^{-1 / p^{i}(p-1)}$ for 
$i \in\{0\} \cup N$ and, $u$ has $N_{i}=p^{i}(p-1) / 2$ zeros on the annulus $\left\{x \in \boldsymbol{Q}_{p}^{\text {alg }} ;|x|=p^{-1 / p^{i}(p-1)}\right\}$ of radius $r_{i}=p^{-1 / p^{i}(p-1)}$ for $i \in\{0\} \cup N$. By Proposition 3.1, $u$ satisfies Proposition 3.9 (ii). Therefore, $u$ satisfies $\left(A_{1}\right)$. For $r \in\left[p^{-1 /(p-1)}, 1\right)$, fix the largest integer $n$ such that $p^{-1 / p^{n}(p-1)}<r$. Then

$$
\begin{aligned}
\log _{p}|u|_{r}-\log _{p}\left|u^{\phi}\right|_{r} \\
=\left(K(r, v)-K\left(r, v^{\phi}\right)\right) \log _{p} r-\sum_{i=0}^{n} N_{i} \log _{p} r_{i}+\sum_{i=0}^{n-1} p N_{i} \log _{p} r_{i}^{1 / p} \\
=\left(\sum_{i=0}^{n} N_{i}-\sum_{i=0}^{n-1} p N_{i}\right) \log _{p} r-\sum_{i=0}^{n} \frac{p^{i}(p-1)}{2} \log _{p} p^{-1 / p^{i}(p-1)} \\
\quad+\sum_{i=0}^{n-1} \frac{p^{i+1}(p-1)}{2} \log _{p} p^{-1 / p^{i+1}(p-1)} \\
=\frac{p-1}{2} \log _{p} r+\frac{1}{2} .
\end{aligned}
$$

Therefore, $u$ is exactly of log-growth $1 / 2$ by Proposition 3.8. Moreover, Tsuzuki computed the corresponding Frobenius slope is $1 / 2$.

4. Special cases. Let $f$ be a nonzero element of $\mathcal{R}$ which satisfies a Frobenius equation

$$
a_{0} f+a_{1} f^{\phi}+\cdots+a_{n} f^{\phi^{n}}=0\left(a_{i} \in \mathcal{R}_{0} a_{0} \neq 0\right) .
$$

Assume $a_{0}, \ldots, a_{n}, f \in \mathcal{A}\left[r_{0}^{p^{n}}, 1\right)$. We define the symbols

$$
\begin{aligned}
I_{i j} & =\left\{r \in\left[r_{0}, 1\right) ;\left|a_{i} f^{\phi^{i}}\right|_{r}=\left|a_{j} f^{\phi^{j}}\right|_{r}=\sup _{k}\left|a_{k} f^{\phi^{k}}\right|_{r}\right\}, \\
I_{i} & =\left\{r \in\left[r_{0}, 1\right) ;\left|a_{i} f^{\phi^{i}}\right|_{r}=\sup _{k}\left|a_{k} f^{\phi^{k}}\right|_{r}\right\}, \\
I_{i}(r) & =I_{i} \cap[r, 1), \quad I_{i j}(r)=I_{i j} \cap[r, 1) .
\end{aligned}
$$

Since $f$ satisfies (13), we have

$$
\bigcup_{0 \leq i<j \leq n} I_{i j}=\left[r_{0}, 1\right) .
$$

The aim of this section is to prove the following theorem (see [4, Proposition 7.6] in the case of rank 2).

THEOREM 4.1. Let $f$ be a nonzero element of the Robba ring $\mathcal{R}$ which satisfies a Frobenius equation

$$
a_{0} f+a_{1} f^{\phi}+a_{2} f^{\phi^{2}}+\cdots+a_{n} f^{\phi^{n}}=0 \quad\left(a_{i} \in \mathcal{R}_{0} a_{0} \neq 0\right) .
$$

Suppose that $a_{0}, \ldots, a_{n}$ satisfy the condition

(a) $\left|a_{1} / a_{0}\right|_{1}>\left|a_{2} / a_{1}\right|_{1}>\cdots>\left|a_{n} / a_{n-1}\right|_{1}$. 
Then $f$ satisfies $\left(A_{1}\right)$. Moreover, $f$ is exactly of log-growth $\log _{p}\left|a_{i+1} / a_{i}\right|_{1}$ for some $i$.

To prove Theorem 4.1, firstly we prove the following lemma.

LEMMA 4.2. Let $f$ be a nonzero element of the Robba ring $\mathcal{R}$ which satisfies a Frobenius equation (13). Assume

$$
\left|a_{1} / a_{0}\right|_{1}>\max _{n-1 \geq i \geq 1}\left|a_{i+1} / a_{i}\right|_{1} .
$$

Then there exists a real number $R_{0} \in[0,1)$ such that $r \in I_{0}\left(R_{0}\right)$ implies $r^{1 / p} \in I_{01}$.

PROOF. By definition, there exists a real number $r_{0}$ such that $a_{0}, \ldots, a_{n}, f \in \mathcal{A}\left[r_{0}^{p^{n}}, 1\right)$. By assumption, we obtain

$$
\left|a_{i} / a_{0}\right|_{1}>\left|a_{i+1} / a_{1}\right|_{1} \quad(i=1, \ldots, n-1) .
$$

Since each $a_{k}$ is bounded, Lemma 2.6 implies that there exists a real number $r_{i}$ such that

$$
\left|a_{i} / a_{0}\right|_{r}>\left|a_{i+1} / a_{1}\right|_{r^{1 / p}} \quad(i=1, \ldots, n-1)
$$

for all $r \in\left[r_{i}, 1\right)$. Put $R_{0}=\max \left\{r_{i}\right\}$ and take $r \in I_{0}\left(R_{0}\right)$. Then we have

$$
\begin{aligned}
\log \left|f^{\phi}\right|_{r^{1 / p}}-\log \left|f^{\phi^{i+1}}\right|_{r^{1 / p}} & =\log |f|_{r}-\log \left|f^{\phi^{i}}\right|_{r} \\
& \geq \log \left|a_{i} / a_{0}\right|_{r} \\
& >\log \left|a_{i+1} / a_{1}\right|_{r^{1 / p}}
\end{aligned}
$$

for $i=1, \ldots, n-1$. Therefore, $\left|a_{1} f^{\phi}\right|_{r^{1 / p}}>\left|a_{i+1} f^{\phi^{i+1}}\right|_{r^{1 / p}}$ for $i=1, \ldots, n-1$. By the equation (14), we conclude $r^{1 / p} \in I_{01}$.

The same argument implies the following lemma.

LEMMA 4.3. Let $f$ be a nonzero element of the Robba ring $\mathcal{R}$ which satisfies a Frobenius equation (13). We also assume

$$
\min _{n-2 \geq i \geq 0}\left|a_{i+1} / a_{i}\right|_{1}>\left|a_{n} / a_{n-1}\right|_{1} .
$$

Then there exists a real number $R_{0} \in[0,1)$ such that $r \in I_{n}\left(R_{0}\right)$ implies $r^{p} \in I_{n-1, n}$.

PRoOF. There exists a real number $r_{0}$ such that $a_{0}, \ldots, a_{n}, f \in \mathcal{A}\left[r_{0}^{p^{n}}, 1\right)$. By assumption, we have

$$
\left|a_{n-1} / a_{i}\right|_{1}>\left|a_{n} / a_{i+1}\right|_{1}(i=0, \ldots, n-2) .
$$

Since $a_{k} \in \mathcal{R}_{0}$, by Lemma 2.6, there exists a real number $r_{i}$ such that for all $r \in\left[r_{i}, 1\right)$,

$$
\left|a_{n-1} / a_{i}\right|_{r^{p}}>\left|a_{n} / a_{i+1}\right|_{r} .
$$

We will see that we can take $R_{0}=\max \left\{r_{i}^{1 / p}\right\}$. Suppose there exists $r \in I_{n}\left(R_{0}\right)$. Then we have

$$
\begin{aligned}
\log \left|f^{\phi^{i}}\right|_{r p}-\log \left|f^{\phi^{n-1}}\right|_{r p} & =\log \left|f^{\phi^{i+1}}\right|_{r}-\log \left|f^{\phi^{n}}\right|_{r} \\
& \leq \log \left|a_{n} / a_{i+1}\right|_{r} \\
& <\log \left|a_{n-1} / a_{i}\right|_{r p}
\end{aligned}
$$


for $i=0, \ldots, n-2$. Therefore, $\left|a_{n-1} f^{\phi^{n-1}}\right|_{r^{p}}>\left|a_{i} f^{\phi^{i}}\right|_{r^{p}}$ for $i=0, \ldots, n-2$. By equality (14), we conclude $r^{p} \in I_{n-1, n}$.

LEMMA 4.4. Let $f$ be a nonzero element of the Robba ring $\mathcal{R}$ which satisfies a Frobenius equation (13) and assume that the condition (a) of Theorem 4.1.

(i) If $I_{0}\left(R_{0}\right)=I_{1}\left(R_{0}\right)=\cdots=I_{k-1}\left(R_{0}\right)=\emptyset$ for some $R_{0} \in[0,1)$, then there exists $R_{1} \in\left[R_{0}, 1\right)$ such that $r \in I_{k}\left(R_{1}\right)$ implies $r^{1 / p} \in I_{k, k+1}$.

(ii) If $I_{n}\left(R_{0}\right)=I_{n-1}\left(R_{0}\right)=\cdots=I_{k+1}\left(R_{0}\right)=\emptyset$ for some $R_{0} \in[0,1)$, then there exists $R_{1} \in\left[R_{0}, 1\right)$ such that $r \in I_{k}\left(R_{1}\right)$ implies $r^{p} \in I_{k-1, k}$.

PROOF. (i) The condition (a) implies $\left|a_{k+1} / a_{k}\right|_{1}>\max _{n-1 \geq i \geq k+1}\left|a_{i+1} / a_{i}\right|_{1}$. We can also show the lemma by the same augments.

By Lemma 4.4, we can calculate the order of log-growth under the condition (a) of Theorem 4.1.

PROPOSITION 4.5. Let $f$ be a nonzero element of the Robba ring $\mathcal{R}$ which satisfies a Frobenius equation (13) and assume that the condition (a) of Theorem 4.1 holds.

(i) If $I_{0}\left(R_{0}\right)=I_{1}\left(R_{0}\right)=\cdots=I_{k-1}\left(R_{0}\right)=\emptyset$ for some $R_{0} \in[0,1)$ and $I_{k}(r) \neq \emptyset$ for any $r \in\left[R_{0}, 1\right)$, then $f$ is exactly of log-growth $\log _{p}\left|a_{k+1} / a_{k}\right|_{1}$.

(ii) If $I_{n}\left(R_{0}\right)=I_{n-1}\left(R_{0}\right)=\cdots=I_{k+1}\left(R_{0}\right)=\emptyset$ for some $R_{0} \in[0,1)$ and $I_{k}(r) \neq \emptyset$ for any $r \in\left[R_{0}, 1\right)$, then $f$ is of log-growth $\log _{p}\left|a_{k} / a_{k-1}\right|_{1}$.

Proof. (i) We prove the assertion in the case where $k=0$. For a general $k$, the similar proof works. By definition, there exists a real number $r_{0}$ such that $a_{0}, \ldots, a_{n}, f, f^{\phi}, \ldots$, $f^{\phi^{n}} \in \mathcal{A}\left[r_{0}, 1\right)$. By Lemma 2.6, there is a real number $r_{1} \in[0,1)$ such that $\left|a_{1} / a_{0}\right|_{s}=p^{t} s^{k}$ with some $t \in \boldsymbol{Q}$ and $k \in \boldsymbol{Z}$ for any $s \in\left[r_{1}, 1\right)$. We fix $r \in I_{0}\left(\max \left\{r_{0}, r_{1}\right\}\right)$. Using Lemma 4.4, we have $r^{1 / p^{n}} \in I_{01}$ for $n>0$. Therefore, we have

$$
\begin{aligned}
|f|_{r^{1 / p}} & =\frac{\left|a_{1}\right|_{r^{1 / p}}}{\left|a_{0}\right|_{r^{1 / p}}}\left|f^{\phi}\right|_{r^{1 / p}}=p^{t} r^{k / p}\left|f^{\phi}\right|_{r^{1 / p}}=p^{t} r^{k / p}|f|_{r}, \\
|f|_{r^{1 / p^{2}}} & =\frac{\left|a_{1}\right|_{r^{1 / p^{2}}}}{\left|a_{0}\right|_{r^{1 / p^{2}}}}\left|f^{\phi}\right|_{r^{1 / p^{2}}}=p^{t} r^{k / p^{2}}\left|f^{\phi}\right|_{r^{1 / p^{2}}}=p^{t} r^{k / p^{2}}|f|_{r^{1 / p}}, \\
& \vdots \\
|f|_{r^{1 / p^{n}}} & =\frac{\left|a_{1}\right|_{r^{1 / p^{n}}}}{\left|a_{0}\right|_{r^{1 / p^{n}}}}\left|f^{\phi}\right|_{r^{1 / p^{n}}}=p^{t} r^{k / p^{n}}\left|f^{\phi}\right|_{r^{1 / p^{n}}}=p^{t} r^{k / p^{n}}|f|_{r^{1 / p^{n-1}}} .
\end{aligned}
$$

By these equalities, we see

$$
|f|_{r^{1 / p^{n}}}=p^{n t} r^{k\left(1 / p+1 / p^{2}+\cdots+1 / p^{n}\right)}|f|_{r} .
$$

Since

$$
r^{k\left(1 / p+1 / p^{2}+\cdots+1 / p^{n}\right)} \rightarrow r^{k /(p-1)}>0(n \rightarrow \infty),
$$

we have

$$
|f|_{r^{1 / p^{n}}}=O\left(p^{t n}\right)=O\left(\log ^{-t}(1 / r)^{1 / p^{n}}\right)
$$


and

$$
|f|_{r^{1 / p^{n}}} \neq o\left(\log ^{-t}(1 / r)^{1 / p^{n}}\right) .
$$

By Proposition 2.9, $f$ is exactly of $\log$-growth $t=\log _{p}\left|a_{1}\right|_{1} /\left|a_{0}\right|_{1}$.

(ii) We note that $f$ and $f^{\phi}$ have the same exact order of log-growth. By the assumption and Lemma 4.4 (2), there exists a real number $r_{0}$ which satisfies the following properties:

- $I_{n}\left(r_{0}\right)=I_{n-1}\left(r_{0}\right)=\cdots=I_{k+1}\left(r_{0}\right)=\emptyset$, and $I_{k}(r) \neq \emptyset$ for any $r \in\left[r_{0}, 1\right)$,

- $a_{0}, \ldots, a_{n}, f, \ldots, f^{\phi^{n}} \in \mathcal{A}\left[r_{0}, 1\right)$,

- $\left|a_{k} / a_{k-1}\right|_{r}=p^{t} r^{s}$ for all $r \in\left[r_{0}, 1\right)$,

- if $r \in I_{k}\left(r_{0}\right)$, then $r^{p} \in I_{k-1, k}$.

By assumption and Lemma 4.4 (ii), there exists a sequence $\left\{t_{n}\right\}_{n \in N}$ which satisfies the following conditions:

- $t_{n} \in I_{k-1, k}$,

- $t_{1} \in\left[r_{0}, 1\right)$ and $t_{n} \in\left(t_{n-1}, 1\right)$ if $n \geq 2$,

- $t_{n} \rightarrow 1(n \rightarrow \infty)$.

For $n \in N$, there exists $m=m(n) \in N \cup\{0\}$ such that

$$
r_{0}^{1 / p^{m}} \leq t_{n} \leq r_{0}^{1 / p^{m+1}}
$$

By the definition of $r_{0}$ and $t_{n}$, we have $t_{n}^{p^{i}} \in I_{k-1, k}$ for $i=0, \ldots, m$. By the definition of $I_{k-1, k}$, we see $\left|a_{k-1} f^{\phi^{k-1}}\right|_{t_{n}^{p^{i}}}=\left|a_{k} f^{\phi^{k}}\right|_{t_{n}^{p^{i}}}$ for $i=0, \ldots, m$. Since $r_{0} \leq t_{n}^{p^{i}}$, we obtain

$$
\left|f^{\phi^{k-1}}\right|_{t_{n}^{p^{i}}}=t_{n}^{s p^{i}} p^{t}\left|f^{\phi^{k}}\right|_{t_{n}^{p^{i}}}=t_{n}^{s p^{i}} p^{t}\left|f^{\phi^{k-1}}\right|_{t_{n}^{p^{i+1}}}
$$

for $i=0, \ldots, m$. Therefore,

$$
\left|f^{\phi^{k-1}}\right|_{t_{n}}=t_{n}^{s\left(1+p+p^{2}+\cdots+p^{m}\right)} p^{t(m+1)}\left|f^{\phi^{k-1}}\right|_{t_{n}^{p^{m+1}}} .
$$

Since we may assume $|f|_{r}$ is an increasing function, we have the formula of inequalities

$$
\begin{aligned}
\left|f^{\phi^{k-1}}\right|_{r_{0}^{1 / p^{m}}} & \leq\left|f^{\phi^{k-1}}\right|_{t_{n}}=t_{n}^{s\left(1+p+p^{2}+\cdots+p^{m}\right)} p^{t(m+1)}\left|f^{\phi^{k-1}}\right|_{t_{n}^{p^{m+1}}} \\
& \leq r_{0}^{-|s|_{\infty}\left(1+1 / p+\cdots+1 / p^{m}\right)} p^{t(m+1)}\left|f^{\phi^{k-1}}\right|_{r_{0}}=O\left(p^{t(m+1)}\right)
\end{aligned}
$$

by (15). Since $m(n) \rightarrow \infty$ as $n \rightarrow \infty$, we conclude that $f$ is of log-growth $t=$ $\log _{p}\left|a_{k} / a_{k-1}\right|_{1}$.

Proof of THEOREM 4.1. There exists integers $k_{1}, k_{2}$ with $1 \leq k_{1} \leq k_{2} \leq n-1$ such that $I_{0}\left(R_{0}\right)=I_{1}\left(R_{0}\right)=\cdots=I_{k_{1}-1}\left(R_{0}\right)=\emptyset, I_{k_{1}}(r) \neq \emptyset, I_{n}\left(R_{0}\right)=I_{n-1}\left(R_{0}\right)=\cdots=$ $I_{k+1}\left(R_{0}\right)=\emptyset, I_{k_{2}}(r) \neq \emptyset$. Then by Proposition 4.5 (i), $f$ is exactly of loggrowth $\log _{p}\left|a_{k_{1}+1} / a_{k_{1}}\right|_{1}$. On the other hand, by Proposition 4.5 (ii), $f$ is of log-growth $\log _{p}\left|a_{k_{2}+1} / a_{k_{2}}\right|{ }_{1}$. Thus we must have $k_{1}=k_{2}$. Since there exists a real number $r \in[0,1)$ such that $I_{k_{1}, k_{1}+1}(r)=[r, 1), f$ satisfies $\left(A_{1}\right)$. 
COROLLARY 4.6. Let $f$ be a nonzero element of $\mathcal{R}$ which satisfies a Frobenius equation (13) and the condition (a) of Theorem 4.1. Let $i$ be an integer such that $f$ is exactly of log-growth $\log _{p}\left|a_{i+1} / a_{i}\right|_{1}$. Then $K\left(1, a_{i+1} / a_{i}\right)$ is divided by $p^{i}$.

PROOF. With the hypothesis of Theorem 4.1, suppose $f$ is exactly of log-growth $\log _{p}\left|a_{i+1} / a_{i}\right|_{1}$ for some $i$ and $\left|a_{i} f^{\phi^{i}}\right|_{r}=\left|a_{i+1} f^{\phi^{i+1}}\right|_{r}$ for any $r$ close enough to 1 . If we put

$$
\log |f|_{r}-\log \left|f^{\phi}\right|_{r}=A(r) \log r+B(r),
$$

then $K\left(r, a_{i+1} / a_{i}\right)=p^{i} A(r)$ is divided by $p^{i}$.

Let $\vec{y}=\left(y_{1}, y_{2}, \ldots, y_{n}\right)$ be a nonzero element of $\mathcal{R}^{n}$. Set the exact order of log-growth of $y_{i}$ by $\alpha_{i}$. We define the exact order of log-growth of $\vec{y}$ to be $\alpha=\max \left\{\alpha_{i}\right\}$. Let $\vec{y}=$ $\left(y_{1}, y_{2}, \ldots, y_{n}\right)$ be a nonzero element of $\mathcal{R}^{n}$ such that

$$
\left(\begin{array}{cccc}
b_{1} & 1 & & \\
\vdots & & \ddots & \\
b_{n-1} & & & 1 \\
b_{n} & & &
\end{array}\right)\left(\begin{array}{c}
y_{1} \\
\vdots \\
y_{n-1} \\
y_{n}
\end{array}\right)^{\phi}=\gamma\left(\begin{array}{c}
y_{1} \\
\vdots \\
y_{n-1} \\
y_{n}
\end{array}\right)
$$

where $b_{1}, \ldots, b_{n} \in \mathcal{R}_{0}$, and $\gamma \in K$ with $|\gamma|=p^{\lambda}$. Then, according to [4, Lemma 6.18], the exact order of log-growth of $\vec{y}$ equals to the exact order of log-growth of $y_{1}$ and $y_{1}$ satisfies the Frobenius equation

$$
\frac{b_{n}^{\phi^{n-1}}}{\gamma^{n-1}} y_{1}^{\phi^{n}}+\frac{b_{n-1}^{\phi^{n-2}}}{\gamma^{n-2}} y_{1}^{\phi^{n-1}}+\cdots+\frac{b_{1}^{\phi}}{\gamma} y_{1}^{\phi}=y_{1} .
$$

COROLLARY 4.7. With the above condition, assume the first slope of the Newton polygon associated to the polynomial

$$
1+b_{1} X+b_{2} X^{2}+\cdots+b_{n} X^{n}
$$

is $\alpha$. Then $y_{1}$ is of log-growth $\lambda-\alpha$. Moreover, we assume $\log _{p}\left|b_{1}\right|_{1}<\log _{p}\left|b_{2} / b_{1}\right|_{1}<$ $\cdots<\log _{p}\left|b_{n} / b_{n-1}\right|_{1}$. Then the exact order of log-growth of $f$ is one of $\lambda+\log _{p}\left|b_{1}\right|_{1}, \lambda+$ $\log _{p}\left|b_{2} / b_{1}\right|_{1}, \ldots, \lambda+\log _{p}\left|b_{n} / b_{n-1}\right|_{1}$. If the exact order of log-growth of $f$ is $\lambda+$ $\log _{p}\left|b_{i+1} / b_{i}\right|_{1}$, then $K\left(1, b_{i}\right)$ is divided by $p$. In particular, if $K\left(1, b_{j}\right)$ is not divided by $p$ for all $j$, then $y_{1}$ is exactly of log-growth $\lambda-\alpha$.

Proof. By [4, Lemma 6.18], the first slope of the Newton polygon of the polynomial

$$
\frac{b_{n}^{\phi^{n-1}}}{\gamma^{n-1}} X^{n}+\frac{b_{n-1}^{\phi^{n-2}}}{\gamma^{n-2}} X^{n-1}+\cdots+\frac{b_{1}^{\phi}}{\gamma} X+1
$$

is $\alpha-\lambda$. Hence, $y_{1}$ is of $\log$-growth $\lambda-\alpha$ by Theorem 2.10. Assume $\log _{p}\left|b_{1}\right|_{1}<$ $\log _{p}\left|b_{2} / b_{1}\right|_{1}<\cdots<\log _{p}\left|b_{n} / b_{n-1}\right|_{1}$. By [4, Lemma 6.18], the slopes of the Newton polygon of polynomial (16) are $-\log _{p}\left|b_{1}\right|_{1}-\lambda>-\log _{p}\left|b_{2} / b_{1}\right|_{1}-\lambda>\cdots>-\log _{p}\left|b_{n} / b_{n-1}\right|_{1}-$

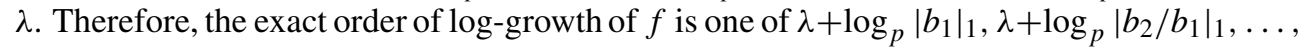
$\lambda+\log _{p}\left|b_{n} / b_{n-1}\right|_{1}$ by Theorem 4.1. Moreover, assume $f$ is exactly of log-growth $\lambda+$ 
$\log _{p}\left|b_{i+1} / b_{i}\right|_{1}$ for $i \geq 1$. By Corollary 4.6, $\lim _{r \rightarrow 1^{-}} K\left(r, b_{i+1}^{\phi^{i}} / b_{i}^{\phi^{i-1}}\right)=\lim _{r \rightarrow 1^{-}}$ $\left(K\left(r, b_{i+1}^{\phi^{i}}\right)-K\left(r, b_{i}^{\phi^{i-1}}\right)\right)$ is divided by $p^{i}$. Since $\lim _{r \rightarrow 1^{-}} K\left(r, b_{i+1}^{\phi^{i}}\right)$ is divided by $p^{i}$ and $\lim _{r \rightarrow 1^{-}} K\left(r, b_{i}^{\phi^{i-1}}\right)$ is divided by $p^{i-1}$ by Proposition $3.1, \lim _{r \rightarrow 1^{-}} K\left(r, b_{i}\right)$ is divided by $p$.

\title{
REFERENCES
}

[ 1 ] Y. ANDRÉ, Dwork's conjecture on the logarithmic growth of solutions of $p$-adic differential equations, Compos. Math. 144 (2008), 484-494.

[2] A. BoutabaA And A. Escassut, Urs and ursims for $p$-adic meromorphic functions inside a disc, Proc. Edinb. Math. Soc. (2) 44 (2001), 485-504.

[ 3 ] W. CHERry, Lectures on Non-Archimedean Function Theory, preprint (2009), math. CV/0909:4590.

[ 4 ] B. Chiarellotto AND N. TsuZUKi, Logarithmic growth and Frobenius filtrations for solutions of $p$-adic differential equations, J. Inst. Math. Jussieu (2009), 465-505.

[ 5 ] B. Chiarellotto And N. Tsuzuki, Log-growth filtration and Frobenius slope filtration of $F$-isocrystals at the generic point and special point, Documenta Math. 16 (2011), 33-64.

[6] G. Christol, Modules différentiels et équations différentielles p-adiques, Queen's Papers in Pure and Appl. Math. 66, Queen's University, Kingston, ON, 1983.

[ 7 ] R. CREw, $F$-isocrystals and $p$-adic representations, Algebraic geometry, Bowdoin, 1985 (Brunswick, Maine, 1985), 111-138, Proc. Sympos. Pure Math. 46, Amer. Math. Soc, Providence, RI, 1987.

[ 8 ] B. M. DwORK, On p-adic differential equations II, Ann. of Math. (2) 98 (1973), 366-376.

[9] B. M. DWORK, Lectures on $p$-adic differential equations, Grundlehren Math. Wiss. 253, Springer-Verlag, New York-Berlin, 1982.

[10] P.-C. HU AND C.-C. YANG, Meromorphic functions over non-Archimedean fields, Math. Appl. 522, Kluwer Academic Publishers, Dordrecht, 2000.

[11] K. Kedlaya, $p$-adic differential equations, Cambridge Stud. Adv. Math.125, Cambridge University Press, Cambridge 2010.

[12] S. Mats UdA, Local indices of $p$-adic differential operators corresponding to Artin-Schreier-Witt coverings, Duke Math. J. 77 (1995), 607-625.

[13] P. Robba And G. Christol, Équation différentielles $p$-adiques, Application aux sommes exponentielles, Actualite Math. Hermann, Paris, 1994.

[14] P. RoвbA, On the index of p-adic differential operators I, Ann. of Math. (2) 101 (1975), 280-316.

[15] A. Robert, A course in $p$-adic analysis, Grad. Texts in Math. 198, Springer-Verlag, New York, 2000.

[16] N. TsUZUKI, Slope filtration of quasi-unipotent overconvergent $F$-isocrystals, Ann. Inst. Fourier (Grenoble) 48 (1998), 379-412.

\author{
DEPARTMENT OF MATHMATICS AND INFORMATICS FACULTY \\ OF SCIENCES CHIBA UNIVERSITY \\ 1-33 YAYOI-CHO, INAGE, CHIBA 263-8522 \\ JAPAN \\ E-mail address: tnakagaw@g.math.s.chiba-u.ac.jp
}

